\title{
$\alpha$-thalassaemia in Apulia: biosynthetic studies
}

\author{
G GUANTi*, A LONOCE*, A Pietrapertosa $\dagger$, G POlimeno $\dagger$, \\ AND N TANNOIA $\dagger$ \\ From *Istituto di Genetica and †Istituto di Clinica Medica I, Università di Bari, Bari, Italy.
}

SUMMARY Analysis of haemoglobin chain synthesis was performed in 15 Apulian patients with $\mathrm{Hb} \mathrm{H}$ disease and in their parents and offspring. The Apulian carriers of $\mathrm{Hb} \mathrm{H}$ disease show a marked imbalance of $\alpha$ and $\beta$ chain synthesis $(0 \cdot 39 \pm 0 \cdot 1)$ with variable clinical and haematological manifestations. However, we are dealing with an intermediate form similar to that described in Italians from other regions. A significant difference was found between the mean $\alpha / \beta$ ratio values $(0 \cdot 81 \pm 0 \cdot 13)$ of parents and offspring of $\mathrm{Hb} \mathrm{H}$ patients and those of the normal controls $(1 \cdot 05 \pm$ $0 \cdot 09$ ); however, extensive overlapping between these two groups exists.

These results have led us to the conclusion that the forms of $\alpha$-thalassaemia found in Apulia are similar to the $\alpha$ defects observed in Sicily; in both cases, in fact, haemoglobin chain synthesis was an unreliable test for discriminating between $\alpha$-thalassaemia- 1 trait and $\alpha$-thalassaemia- 2 trait.

The $\alpha$-thalassaemia syndromes are inherited anaemias caused by defective function of one or more $\alpha$ globin genes. In most human populations, ${ }^{12}$ $\alpha$ globin structural gene loci are duplicated so that each diploid cell contains four copies of $\alpha$ globin genes. Four main $\alpha$-thalassaemia syndromes of increasing clinical severity are recognised: (1) the silent carrier state ( $\alpha$-thalassaemia-2) with no clinical manifestations; (2) $\alpha$-thalassaemia trait $(\alpha$-thalassaemia-1) characterised by microcytic red blood cells but little or no anaemia; (3) haemoglobin $\mathbf{H}$ disease, which manifests as haemolytic anaemia; and (4) homozygous $\alpha$-thalassaemia in which the affected fetus dies at or around term from hydrops fetalis. These four syndromes are the consequence of dysfunctional expression of from one to all four $\alpha$ globin genes, respectively.

Patients with $\alpha$-thalassaemia are usually of Asian, Mediterranean, or Negro descent. Gene counting experiments indicate that one or more $\alpha$ globin genes are deleted in blacks ${ }^{3}{ }^{4}$ and in most ${ }^{4-6}$ but not all Asians ${ }^{4} 7$ with $\alpha$-thalassaemia. By restriction endonuclease patterns the presence of deletions as well as more complex defects have been demonstrated $^{8}$ in people of Mediterranean origin. These observations suggest that $\alpha$-thalassaemia may be heterogeneous among people of different ethnic backgrounds. In this paper, in order to define the molecular characteristics of the dysfunctional expression of the $\alpha$ globin genes in the population of Apulia, as a first approach we investigated the

Received for publication 3 September 1982. Accepted for publication 14 October 1982. globin chain synthesis in families of patients with $\mathrm{Hb} \mathbf{H}$ disease, observed by one of us $^{9}$ during the last few years.

\section{Materials and methods}

The families described here were selected for globin chain biosynthetic studies whenever a patient with $\mathrm{Hb} \mathrm{H}$ was found.

Routine haematological investigations were carried out by standard methods. Full blood counts were made with the Coulter Counter. Haemoglobin electrophoresis was performed on cellulose acetate strips at $\mathrm{pH} 8 \cdot 6$. The different fractions were measured after elution of the bands and read spectrophotometrically at $415 \mathrm{~nm}$. Haemoglobin $A_{2}$ was quantified by DE 52 microchromatography and $\mathrm{Hb} \mathrm{F}$ was determined by alkali denaturation according to the method of Betke et al..$^{10}$ Preparations for detection of $\mathrm{Hb} \mathrm{H}$ inclusion bodies were made after incubation of equal volumes of blood and $1 \%$ Brilliant Cresyl Blue at $37^{\circ} \mathrm{C}$ for 1 to 2 hours. Red cell osmotic fragility was assessed according to Silvestroni and Bianco. ${ }^{11}$ Serum iron and iron binding capacity was determined by the method of Lauber. ${ }^{12}$

Globin chain synthesis was performed by incubation of washed red cells $(0.8 \mathrm{ml})$ with an equal volume of a leucine free amino-acid reagent mixture, identical to that of Lingrel and Borsook, ${ }^{13}$ except that human dialysed $\mathrm{AB}$ plasma was used in place of rabbit plasma. Addition of Krebs Ringer phosphate buffer (KRP) at $\mathrm{pH} 7.4$ completed the 
incubation mixture which was placed at $37^{\circ} \mathrm{C}$ in a water bath. To this mixture $100 \mu \mathrm{l}{ }^{3} \mathrm{H}$-leucine $(1 \mathrm{mCi} / \mathrm{ml}$, specific activity $130 \mathrm{Ci} / \mathrm{mmol}$, the Radiochemical Centre, Amersham) were added and the cells incubated for 2 hours at $37^{\circ} \mathrm{C}$. Whenever the reticulocyte counts were low $(<1 \%)$ reticulocyte enrichment with Percol gradients was performed. After 2 hours of incubation the cells were washed three times at $4^{\circ} \mathrm{C}$ in KRP to remove free radioactivity and lysed with three volumes of distilled water. The lysate was thawed and added in drops to an acid-acetone mixture. Globin was fractionated on CM cellulose chromatography in $8 \mathrm{~mol} / 1$ urea. ${ }^{14}$ Optical density was continuously monitored by an LKB Uvicord at $280 \mathrm{~nm}$. The radioactivity of each fraction was measured with a Beckman scintillation counter. The total radioactivity incorporated into the $\alpha$ and $\beta$ chains was determined.

\section{Results}

Clinical and haematological data are summarised in tables 1 to 4 . The results of the globin chain biosynthetic studies are illustrated in the figure.

All 15 patients with $\mathrm{Hb} \mathrm{H}$ disease had a decreased $\alpha$ chain synthesis with a mean $\alpha / \beta$ ratio of $0.39 \pm$ $0 \cdot 1$ (table 1 , figure $c$ ). The $\alpha / \beta$ ratio in 24 parents of the patients with $\mathrm{Hb} \mathrm{H}$ disease was $0.79 \pm 0 \cdot 15$ (table 2 , figure $b$ ). The mean $\alpha / \beta$ ratio in the 36 control subjects was close to unity (figure a). Although there was no overlap in the mean $\alpha / \beta$ ratio between carriers of $\alpha$-thalassaemia and controls, at least seven obligate heterozygotes had $\alpha / \beta$ ratios in the normal range.

Offspring of patients with $\mathrm{Hb} \mathrm{H}$ disease, all obligate carriers of $\alpha$-thalassaemia, excluding the cases with parent-child transmission of $\mathrm{Hb} \mathrm{H}$ disease,

TABLE 1 Haematological values in 15 subjects with $H b H$ disease.

\begin{tabular}{|c|c|c|c|c|c|c|c|c|c|c|c|c|c|c|c|}
\hline \multirow{2}{*}{$\begin{array}{l}\text { Case } \\
\text { No } \\
1\end{array}$} & \multirow{2}{*}{$\begin{array}{l}R B C \\
\left(x 10^{12} / l\right)\end{array}$} & \multirow{2}{*}{$\begin{array}{l}\begin{array}{l}H b \\
(g / d l)\end{array} \\
6 \cdot 2\end{array}$} & \multirow{2}{*}{$\begin{array}{l}\begin{array}{l}H t \\
(\%)\end{array} \\
28 \cdot 7\end{array}$} & \multirow{2}{*}{$\begin{array}{l}M C V \\
(f) \\
76\end{array}$} & \multirow{2}{*}{$\begin{array}{l}M C H \\
(p g) \\
16\end{array}$} & \multirow{2}{*}{$\begin{array}{l}M C H C \\
(g / d l) \\
21\end{array}$} & \multirow{2}{*}{$\begin{array}{l}\begin{array}{l}H b A_{2} \\
(\%)\end{array} \\
1 \cdot 5\end{array}$} & \multirow{2}{*}{$\begin{array}{l}\begin{array}{c}H b H \\
(\%)\end{array} \\
14 \cdot 2\end{array}$} & \multicolumn{3}{|c|}{$\begin{array}{l}\text { Inclusion } \\
\text { bodies }\end{array}$} & \multicolumn{3}{|c|}{$\begin{array}{l}\text { Erythrocyte } \\
\text { morphology }\end{array}$} & $\begin{array}{l}\alpha / \beta \\
\text { ratio }\end{array}$ \\
\hline & & & & & & & & & + & + & + & + & + & + & 0.40 \\
\hline 2 & $4 \cdot 91$ & 9.9 & $34 \cdot 8$ & 71 & 20 & 28 & $1 \cdot 9$ & $5 \cdot 0$ & + & + & - & + & + & - & 0.46 \\
\hline 3 & $5 \cdot 80$ & $8 \cdot 5$ & $35 \cdot 0$ & 60 & 14 & 24 & $1 \cdot 8$ & $5 \cdot 0$ & + & + & - & + & + & + & 0.60 \\
\hline 4 & $3 \cdot 75$ & $10 \cdot 0$ & 37.0 & 98 & 36 & 27 & $1 \cdot 4$ & $22 \cdot 0$ & + & + & + & + & + & + & 0.33 \\
\hline 5 & $3 \cdot 20$ & $7 \cdot 0$ & $22 \cdot 0$ & 68 & 21 & 31 & 1.4 & $38 \cdot 0$ & + & + & + & + & - & - & 0.26 \\
\hline 6 & $6 \cdot 10$ & $11 \cdot 4$ & $36 \cdot 0$ & 59 & 18 & 31 & $2 \cdot 6$ & $10 \cdot 0$ & + & + & + & - & - & - & 0.35 \\
\hline 7 & 5.00 & $8 \cdot 0$ & $25 \cdot 0$ & 50 & 16 & 32 & $3 \cdot 4$ & 7.0 & + & + & + & + & + & + & 0.62 \\
\hline 8 & $5 \cdot 20$ & $9 \cdot 2$ & 37.0 & 86 & 18 & 29 & $1 \cdot 2$ & $5 \cdot 0$ & + & + & - & + & + & - & 0.37 \\
\hline 9 & $4 \cdot 80$ & $7 \cdot 1$ & 29.0 & 72 & 19 & 25 & $2 \cdot 2$ & $6 \cdot 1$ & + & + & - & + & - & - & 0.41 \\
\hline 10 & 3.95 & $7 \cdot 3$ & 32.0 & 86 & 24 & 26 & $1 \cdot 9$ & $11 \cdot 1$ & + & + & + & + & + & - & 0.40 \\
\hline 11 & $4 \cdot 58$ & $8 \cdot 1$ & $33 \cdot 0$ & 89 & 22 & 25 & $2 \cdot 7$ & $7 \cdot 5$ & + & + & + & + & + & - & 0.38 \\
\hline 12 & $3 \cdot 54$ & $7 \cdot 6$ & $31 \cdot 2$ & 78 & 23 & 28 & $2 \cdot 5$ & $9 \cdot 5$ & + & + & + & + & + & - & 0.39 \\
\hline 13 & $4 \cdot 00$ & $8 \cdot 1$ & 27.0 & 68 & $23 \cdot 5$ & 26 & $2 \cdot 0$ & $6 \cdot 3$ & + & + & - & + & - & - & 0.36 \\
\hline 14 & 4.95 & $7 \cdot 9$ & 30.0 & 85 & 25 & 27 & $2 \cdot 1$ & $8 \cdot 0$ & + & + & + & + & + & - & 0.26 \\
\hline 15 & $3 \cdot 39$ & $7 \cdot 3$ & $31 \cdot 5$ & 81 & 17 & 25 & $2 \cdot 6$ & $9 \cdot 9$ & + & + & + & + & + & + & 0.30 \\
\hline
\end{tabular}

TABLE 2 Haematological values in parents of $\mathrm{Hb} H$ disease patients.

\begin{tabular}{|c|c|c|c|c|c|c|c|c|c|c|c|c|c|c|}
\hline $\begin{array}{l}\text { Case } \\
\text { No }\end{array}$ & $\begin{array}{l}R B C \\
\left(x 10^{12} / l\right)\end{array}$ & $\begin{array}{l}H b \\
(g / d l)\end{array}$ & $\begin{array}{l}H t \\
(\%)\end{array}$ & $\begin{array}{l}M C V \\
(f)\end{array}$ & $\begin{array}{l}M C H \\
(p g)\end{array}$ & $\begin{array}{l}M C H C \\
(g / d l)\end{array}$ & $\begin{array}{l}H b A_{2} \\
(\%)\end{array}$ & $\begin{array}{l}\text { Inc } \\
\text { boc }\end{array}$ & $\begin{array}{l}\text { usior } \\
\text { ies }\end{array}$ & & & $\begin{array}{l}\text { thro } \\
\text { pho } \\
P\end{array}$ & $\begin{array}{l}\text { ogte } \\
\text { ogy }\end{array}$ & $\begin{array}{l}\alpha / \beta \\
\text { ratio }\end{array}$ \\
\hline 1 & $5 \cdot 73$ & $13 \cdot 3$ & 39.9 & 69 & $23 \cdot 0$ & $33 \cdot 0$ & $1 \cdot 6$ & \pm & - & - & + & + & + & 0.58 \\
\hline 3 & $5 \cdot 51$ & $13 \cdot 2$ & $43 \cdot 7$ & 79 & $23 \cdot 0$ & $30 \cdot 0$ & $1 \cdot 8$ & + & + & - & - & - & - & $1 \cdot 20$ \\
\hline 4 & $4 \cdot 65$ & $13 \cdot 1$ & $38 \cdot 4$ & 82 & $28 \cdot 0$ & $34 \cdot 0$ & $2 \cdot 3$ & \pm & - & - & + & - & - & 0.67 \\
\hline 5 & $6 \cdot 10$ & $15 \cdot 3$ & $50 \cdot 0$ & 81 & $25 \cdot 0$ & $30 \cdot 0$ & $2 \cdot 3$ & + & - & - & + & - & - & 0.70 \\
\hline 6 & $6 \cdot 60$ & $17 \cdot 1$ & $50 \cdot 0$ & 87 & $25 \cdot 0$ & $34 \cdot 0$ & $2 \cdot 7$ & \pm & - & - & + & - & - & 0.72 \\
\hline 8 & $4 \cdot 50$ & $15 \cdot 2$ & $40 \cdot 0$ & 88 & $33 \cdot 0$ & $38 \cdot 0$ & $2 \cdot 6$ & - & - & - & - & - & - & 1.00 \\
\hline 9 & $4 \cdot 50$ & $13 \cdot 0$ & $43 \cdot 0$ & 95 & $28 \cdot 0$ & $30 \cdot 0$ & $2 \cdot 7$ & - & - & - & + & + & - & 0.69 \\
\hline 10 & $3 \cdot 50$ & $11 \cdot 5$ & $37 \cdot 0$ & 105 & $32 \cdot 0$ & $31 \cdot 0$ & $2 \cdot 5$ & - & - & - & + & - & - & 0.81 \\
\hline 11 & $5 \cdot 31$ & $12 \cdot 1$ & $40 \cdot 2$ & 91 & $22 \cdot 1$ & $32 \cdot 1$ & 2.6 & \pm & - & - & + & + & \pm & 0.66 \\
\hline 12 & 4.97 & $11 \cdot 9$ & $37 \cdot 8$ & 88 & $23 \cdot 8$ & $30 \cdot 8$ & 2.4 & - & - & - & + & \pm & \pm & 0.71 \\
\hline 13 & 5.45 & $13 \cdot 2$ & $39 \cdot 7$ & 88 & $32 \cdot 0$ & $33 \cdot 0$ & $2 \cdot 7$ & - & - & - & - & - & $\overline{-}$ & 0.94 \\
\hline 14 & $4 \cdot 99$ & $10 \cdot 8$ & $38 \cdot 2$ & 89 & $30 \cdot 0$ & $34 \cdot 0$ & $2 \cdot 7$ & - & - & - & + & - & - & 0.73 \\
\hline 15 & 5.02 & $13 \cdot 0$ & 37.0 & 87 & $29 \cdot 1$ & $32 \cdot 4$ & $2 \cdot 5$ & - & - & - & + & - & - & 0.90 \\
\hline 16 & $4 \cdot 82$ & $12 \cdot 2$ & $35 \cdot 0$ & 95 & 27.4 & $30 \cdot 1$ & $2 \cdot 6$ & - & - & - & + & \pm & - & 0.83 \\
\hline 18 & $5 \cdot 27$ & $11 \cdot 9$ & $38 \cdot 0$ & 99 & 28.0 & $35 \cdot 0$ & $2 \cdot 7$ & - & - & - & + & - & \pm & 0.80 \\
\hline 19 & $5 \cdot 53$ & $11 \cdot 3$ & $39 \cdot 0$ & 91 & $24 \cdot 0$ & $30 \cdot 0$ & $2 \cdot 2$ & - & - & - & - & - & - & 0.99 \\
\hline 20 & 5.00 & $11 \cdot 6$ & $40 \cdot 0$ & 89 & 27.0 & $32 \cdot 0$ & $2 \cdot 4$ & - & - & - & - & - & - & 0.90 \\
\hline 21 & $5 \cdot 60$ & $11 \cdot 8$ & $38 \cdot 0$ & 85 & 26.0 & 29.9 & $2 \cdot 6$ & \pm & - & - & + & + & - & 0.70 \\
\hline 22 & $5 \cdot 43$ & $10 \cdot 3$ & 37.0 & 89 & $26 \cdot 0$ & $31 \cdot 0$ & $2 \cdot 3$ & \pm & - & - & + & + & + & 0.81 \\
\hline 23 & $5 \cdot 15$ & $12 \cdot 9$ & $40 \cdot 0$ & 88 & $27 \cdot 0$ & $33 \cdot 0$ & $2 \cdot 7$ & - & - & - & - & - & - & 1.00 \\
\hline 24 & $5 \cdot 06$ & $13 \cdot 2$ & $40 \cdot 0$ & 87 & 29.0 & 28.0 & $2 \cdot 2$ & + & - & - & + & + & \pm & 0.66 \\
\hline
\end{tabular}


TABLE 3 Haematological values in seven offspring of $\mathrm{Hb} H$ patients.

\begin{tabular}{|c|c|c|c|c|c|c|c|c|c|c|c|c|c|c|}
\hline $\begin{array}{l}\text { Case } \\
\text { No }\end{array}$ & $\begin{array}{l}R B C \\
\left(x 10^{12} / l\right)\end{array}$ & $\begin{array}{l}H b \\
(g / d l)\end{array}$ & $\begin{array}{l}H t \\
(\%)\end{array}$ & $\begin{array}{l}M C V \\
(f)\end{array}$ & $\begin{array}{l}M C H \\
(p g)\end{array}$ & $\begin{array}{c}M C H C \\
(g / d l)\end{array}$ & $\begin{array}{c}H b A_{2} \\
(\%)\end{array}$ & $\begin{array}{l}\text { Inc } \\
\text { bod }\end{array}$ & $\begin{array}{l}\text { usion } \\
\text { ies }\end{array}$ & & $\begin{array}{l}\text { Ery } \\
\text { mo } \\
\boldsymbol{A}\end{array}$ & $\begin{array}{c}\text { vthro } \\
\text { rpho } \\
P\end{array}$ & $\begin{array}{c}\text { cyte } \\
\operatorname{logy} \\
H\end{array}$ & $\begin{array}{l}\alpha / \beta \\
\text { ratio }\end{array}$ \\
\hline $\begin{array}{l}1 \\
2 \\
3 \\
4 \\
5 \\
6 \\
7\end{array}$ & $\begin{array}{l}4 \cdot 60 \\
5 \cdot 50 \\
5 \cdot 00 \\
6 \cdot 24 \\
5 \cdot 30 \\
6 \cdot 13 \\
4 \cdot 40\end{array}$ & $\begin{array}{l}13.5 \\
16.0 \\
14.7 \\
11.6 \\
13.6 \\
11.7 \\
10.7\end{array}$ & $\begin{array}{l}39.0 \\
47.0 \\
43.0 \\
40.4 \\
41.8 \\
39.8 \\
34.5\end{array}$ & $\begin{array}{l}84 \\
85 \\
68 \\
69 \\
79 \\
65 \\
88\end{array}$ & $\begin{array}{l}29 \cdot 0 \\
29 \cdot 0 \\
29 \cdot 0 \\
28 \cdot 6 \\
25 \cdot 7 \\
19 \cdot 1 \\
30 \cdot 0\end{array}$ & $\begin{array}{l}34 \cdot 0 \\
34 \cdot 0 \\
34 \cdot 0 \\
28 \cdot 7 \\
32 \cdot 5 \\
29 \cdot 4 \\
33 \cdot 0\end{array}$ & $\begin{array}{l}2 \cdot 4 \\
2 \cdot 8 \\
2 \cdot 9 \\
2 \cdot 2 \\
2 \cdot 5 \\
1 \cdot 8 \\
2 \cdot 7\end{array}$ & $\begin{array}{l}- \\
- \\
- \\
- \\
- \\
-\end{array}$ & $\begin{array}{l}- \\
- \\
- \\
- \\
- \\
-\end{array}$ & $\begin{array}{l}- \\
- \\
- \\
- \\
- \\
-\end{array}$ & $\begin{array}{l}- \\
- \\
- \\
- \\
\pm \\
+ \\
-\end{array}$ & $\begin{array}{l}- \\
- \\
- \\
+ \\
+\end{array}$ & $\begin{array}{l}- \\
- \\
- \\
- \\
- \\
-\end{array}$ & $\begin{array}{l}0.83 \\
0.85 \\
0.80 \\
0.87 \\
0.90 \\
0.88 \\
0.92\end{array}$ \\
\hline
\end{tabular}

TABLE 4 Summary of haematological mean values.

\begin{tabular}{|c|c|c|c|c|c|c|c|c|}
\hline & $\begin{array}{l}R B C \\
\left(x 10^{12} l\right)\end{array}$ & $\begin{array}{l}H b \\
(g / d l)\end{array}$ & $\begin{array}{l}H t \\
(\%)\end{array}$ & $\begin{array}{l}M C V \\
(f)\end{array}$ & $\begin{array}{l}M C H \\
(p g)\end{array}$ & $\begin{array}{l}M C H C \\
(g / d l)\end{array}$ & $\begin{array}{l}\mathrm{Hb} \mathrm{A}_{2} \\
(\%)\end{array}$ & $\begin{array}{l}\alpha / \beta \\
\text { ratio }\end{array}$ \\
\hline \multirow[t]{2}{*}{ Normal controls $(n=36)$} & $\begin{array}{l}4 \cdot 46 \pm \\
0 \cdot 20\end{array}$ & $\begin{array}{l}15 \cdot 40 \pm \\
1 \cdot 00\end{array}$ & $\begin{array}{l}44 \cdot 40 \pm \\
3 \cdot 70\end{array}$ & $\begin{array}{l}92 \cdot 71 \pm \\
2 \cdot 50\end{array}$ & $\begin{array}{l}32 \cdot 22 \pm \\
1 \cdot 20\end{array}$ & $\begin{array}{l}34 \cdot 68 \pm \\
2 \cdot 60\end{array}$ & $\begin{array}{l}2 \cdot 69 \pm \\
0 \cdot 11\end{array}$ & $\begin{array}{l}1 \cdot 05 \pm \\
0 \cdot 10\end{array}$ \\
\hline & & & & \multicolumn{2}{|c|}{$\begin{array}{l}\text { Normal vs } \\
\alpha \text {-thalassaemia trait }\end{array}$} & \multicolumn{2}{|c|}{$\begin{array}{l}t=8.950 \\
p<0.001\end{array}$} & \\
\hline \multirow[t]{2}{*}{$\begin{array}{l}\alpha \text {-thalassaemia trait } \\
(\alpha \text {-thal-1 and } \alpha \text {-thal-2) } \\
(n=31)\end{array}$} & $\begin{array}{l}5 \cdot 15 \pm \\
0.66\end{array}$ & $\begin{array}{c}12 \cdot 70 \pm \\
1 \cdot 60\end{array}$ & $\begin{array}{c}39 \cdot 93 \pm \\
3 \cdot 76\end{array}$ & $\begin{array}{c}85 \cdot 00 \pm \\
8 \cdot 81\end{array}$ & $\begin{array}{c}27 \cdot 04 \pm \\
3 \cdot 18\end{array}$ & $\begin{array}{c}31 \cdot 96 \pm \\
2 \cdot 28\end{array}$ & $\begin{array}{l}2 \cdot 43 \pm \\
0 \cdot 30\end{array}$ & $\begin{array}{l}0.81 \pm \\
0.13\end{array}$ \\
\hline & & & & \multicolumn{2}{|c|}{$\begin{array}{l}\alpha \text {-thalassaemia trait } \\
\text { vs } \mathrm{Hb} \mathrm{H} \text { disease }\end{array}$} & \multicolumn{2}{|c|}{$\begin{array}{l}t=10 \cdot 378 \\
p<0.001\end{array}$} & \\
\hline Hb $\mathbf{H}$ disease & $\begin{array}{l}4 \cdot 46 \pm \\
0 \cdot 89\end{array}$ & $\begin{array}{l}8 \cdot 24 \pm \\
1 \cdot 37\end{array}$ & $\begin{array}{c}31 \cdot 30 \pm \\
4 \cdot 43\end{array}$ & $\begin{array}{l}75 \cdot 13 \pm \\
12 \cdot 93\end{array}$ & $\begin{array}{c}20 \cdot 83 \pm \\
5.53\end{array}$ & $\begin{array}{c}27.00 \pm \\
2.95\end{array}$ & $\begin{array}{l}2 \cdot 08 \pm \\
0.60\end{array}$ & $\begin{array}{l}0 \cdot 39 \pm \\
0 \cdot 10\end{array}$ \\
\hline
\end{tabular}

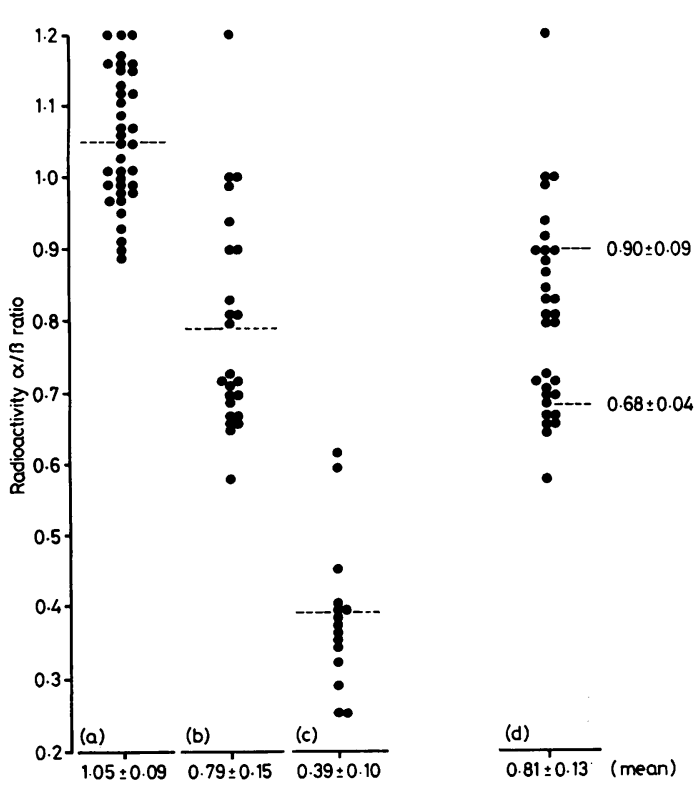

FIG URE Results of globin chain synthesis study in (a) normal controls, (b) parents of patients with $\mathrm{Hb} \mathrm{H}$ disease, $(c)$ patients with $\mathrm{Hb} H$ disease, $(d)$ parents and offspring of $\mathrm{Hb} \mathrm{H}$ potients.

showed a mean $\alpha / \beta$ chain synthesis ratio of $0.86 \pm$ 0.04 (table 3). However, four out of seven did not show alterations of red cell morphology or of $\alpha / \beta$ ratio.

\section{Discussion}

Haemoglobin $\mathbf{H}$ disease in Apulia is characterised by a marked imbalance of $\alpha$ and $\beta$ chain synthesis similar to that observed by Galanello et $a l^{15}$ in Sardinia, Musumeci et al ${ }^{16}$ in Sicily, and Kan et al ${ }^{17}$ in non-Negro patients.

It is well known that carriers of $\mathrm{Hb} \mathbf{H}$ disease show variable clinical and haematological manifestations in different racial groups; the most severe form has been observed in the Far East ${ }^{18}$ and the mild form in American blacks. ${ }^{19}$ In our patients an intermediate form was present, very similar to that described by Galanello $e t a^{15}$ and Musumeci $e t a^{16}$ in Sardinian and Sicilian subjects respectively. None of the families studied showed abnormal haemoglobins like $\mathrm{Hb}$ Constant Spring or $\mathrm{Hb}$ Icaria observed in about $50 \%$ of patients with $\mathrm{Hb} \mathrm{H}$ disease in the Far East, ${ }^{20} 21$ both determined by genes which act like an $\alpha$-thalassaemia-2 gene.

Since $\mathrm{Hb} \mathbf{H}$ disease is known to occur from double heterozygosity for $\alpha$-thalassaemia- 1 and $\alpha$-thalassaemia-2, parents and offspring of patients with this disease must be heterozygous for either $\alpha$-thalassaemia-1 or $\alpha$-thalassaemia-2 traits. Usually one of the parents of a patient with $\mathrm{Hb} \mathrm{H}$ disease has a thalassaemia-like disorder, while the other, the so called 'silent carrier', has either minimal red cell changes or is haematologically normal. Less frequently both parents are affected by the more severe form ( $\alpha$-thalassaemia-1).

According to Kan et $a l^{17}$ and Pootrakul et $a l^{22}$ 
the 'silent carriers' can be detected through the analysis of haemoglobin chain synthesis. In fact, in both of these studies, presumed obligatory carriers of the milder form of $\alpha$-thalassaemia were shown, as a group, to have a mean $\alpha / \beta$ raitio different from both the value of normal controls and the value of the group with the more severe type of disorder. In our case, too, if we pool (figure d) the results of the $\mathrm{Hb}$ chain synthesis observed in parents ( 12 couples) and offspring (seven cases) of patients with $\mathrm{Hb} \mathrm{H}$ disease, the $\alpha / \beta$ ratios segregate, without overlapping, around two different means of $0.90 \pm 0.09$ and $0.68 \pm 0.04$, which could represent the values of $\alpha$-thalassaemia-2 and $\alpha$-thalassaenia-1 traits, respectively. However, the normal $\alpha / \beta$ ratio we found in several silent carriers demonstrates that a carrier of the $\alpha$ thalassaemia- 2 gene will be overlooked by random screening of the population. Furthermore, if the parents of $\mathrm{Hb} \mathrm{H}$ patients are considered one by one, as should be done in every case, it can be seen that in two instances both members of the couple show an $\alpha / \beta$ ratio corresponding to the $\alpha$-thalassaemia-2 trait. Apart from these, we were not able to single out two different groups among the 12 couples of parents of patients with $\mathrm{Hb} \mathrm{H}$ disease.

These data, in agreement with the observations of Musumeci et al ${ }^{16}$ in the Sicilian population, differ from those reported by Pootrakul et al ${ }^{22}$ and Kan et al. ${ }^{17}$

From the clinical point of view, failure to diagnose silent carriers is of no consequence, but different considerations apply to the detection of the silent carrier state for the purpose of genetic counselling. It is also necessary to have a method for the identification of such persons in any population survey to determine the frequency of the $\alpha$-thalassaemia gene.

Further studies of cellular DNA fragments containing the defective $\alpha$ loci and mRNA analysis, according to the recent observations of Orkin and Goff, ${ }^{23}$ could permit the characterisation of specific mutations responsible for abnormal globin gene expression in the different cases.

The authors sincerely thank Dr M Marinucci for helpful advice.

\section{References}

1 Lehmann H, Carrel RW. Differences between $\alpha$ and $\beta$ chain variants of human haemoglobin and between $\alpha$ and $\beta$-thalassaemia. Possible duplication of the $\alpha$-chain gene. Br Med J 1968;4:748-50.

2 Hollan SR, Szelenyi JG, Brimhall B, et al. Multiple alpha chain loci for human haemoglobin: Hb J-Buda and Hb G-Pest. Nature 1972;235:47-50.

3 Davis JR, Dozy AM, Lubin B, et al. Alpha thalassemia in blacks is due to gene deletion. Am J Hum Genet 1979;31: 569-73.

4 Embury SH, Miller JA, Dozy AM, et al. Two different molecular organizations account for the single $\alpha$-globin gene of the $\alpha$-thalassemia-2 genotype. J Clin Invest 1980;66:1319-25.

5 Taylor JM, Dozy AM, Kan YW, et al. Genetic lesion in homozygous $\alpha$-thalassemia (hydrops fetalis). Nature 1974;251:392-3.

${ }^{6}$ Ottolenghi S, Lanyon WG, Paul J, et al. The severe form of $\alpha$ thalassaemia is caused by haemoglobin gene deletion. Nature 1974;251:389-92.

7 Kan YW, Dozy AM, Trecartin R, Todd D. Identification of a non-deletion defect in $\alpha$-thalassemia. $N$ Engl J Med 1977;297:1081-4.

8 Orkin SH, Old J, Lazarus H, et al. The molecular basis of $\alpha$-thalassemias frequent occurrence of dysfunctional loci among non-Asian with $\mathrm{Hb} \mathrm{H}$ disease. Cell 1979;17: $33-42$.

9 Tannoia N, Putignano A. $\alpha$-thalassemia (studio di 4 famiglie). Atti Congresso sulle Microcitemie, Cosenza, 1973:175.

10 Betke K, Marti HR, Schlicht I. Estimation of small percentages of fetal haemoglobin. Nature 1959;184: 1877-8.

11 Silvestroni E, Bianco I. Il metodo di Simmel per lo studio delle resistenze globulari. Policlinico 1945;51:153-5.

12 Lauber K. Determination of serum iron and iron binding capacity without deproteinization. Klin Chem 1965;3: 96-9.

13 Lingrel JB, Borsook H. A comparison of amino acid incorporation into the hemoglobin and ribosomes of marrow erythroid and circulating reticulocytes of severely anemic rabbits. Biochemistry 1963;2:309-14.

14 Clegg JB, Naughton MA, Weatherall DJ. Abnormal human haemoglobins. Separation and characterization of $\alpha$ and $\beta$ chains by chromatography and determination of two new variants, $\mathrm{Hb}$ Chesapeake and $\mathrm{Hb} \mathrm{J}$ (Bangkok). J Mol Biol 1966;19:91-108.

15 Galanello R, Melis MA, Furbetta M, et al. Hemoglobin $\mathbf{H}$ disease in Sardinia: phenotypic and genetic observations. Hemoglobin 1978;2:333-49.

16 Musumeci S, Schilirò G, Pizzarelli G, et al. Alpha thalassaemia in Sicily: haematological and biosynthetic studies. Br J Haematol 1979;43:413-22.

17 Kan JW, Schwartz E, Nathan DG. Globin chain synthesis in the alpha thalassemia syndromes. J Clin Invest 1968; 47:2515-22.

18 Wasi P, Na-Nakorn S, Pootrakul S, et al. Alpha and betathalassemia in Thailand. Ann NY Acad Sci 1969;165: 60-82.

19 Lehmann H, Carrell RW. Variations in the structure of human haemoglobin with particular reference to the mutable haemoglobins. Br Med Bull 1969;25:14-25.

20 Clegg JB, Weatherall DJ. Hemoglobin Constant-Spring: an unusual $\alpha$ chain variant involved in the etiology of hemoglobin H disease. Ann NY Acad Sci 1974;232: 168-78.

21 Clegg JB, Weatherall DJ, Contopoulougriva I, et al. Haemoglobin Icaria a new chain termination mutant which causes $\alpha$-thalassaemia. Nature 1974;251:245-7.

22 Pootrakul S, Sapprapa S, Wasi P, et al. Haemoglobin synthesis in 28 obligatory cases for $\alpha$-thalassaemia traits. Humangenetik 1975;29:121-6.

23 Orkin SH, Goff SC. The duplicated human $\alpha$-globin genes: their relative expression as measured by RNA analysis. Cell $1981 ; 24: 341-51$.

Correspondence and requests for reprints to Professor G Guanti, Istituto di Genetica Università, Via Amendola 165/A, 70126, Bari, Italy. 